\title{
Fronteira entre o uso da Eletrônica baseada em Hardware Livre e Robótica no Contexto Educacional
}

\author{
Rachel C. D. Reis ${ }^{1}$, Larissa F. Rodrigues ${ }^{1}$, Clausius D. G. Reis ${ }^{1}$, Rodrigo Moreira ${ }^{1,2}$ \\ ${ }^{1}$ Universidade Federal de Viçosa, Campus Rio Paranaíba \\ ${ }^{2}$ Universidade Federal de Uberlândia, Campus Santa Mônica \\ \{rachel.reis, larissa.f.rodrigues, clausius, rodrigo\}@ufv.br, \\ rodrigo.moreira@ufu.br
}

\begin{abstract}
The electronic devices, based on free hardware, have been widely used in studies on robotics in the educational context. However, it is observed that some studies confuse the concepts of "electronics" with "robotics". Therefore, this work aims at developing a systematic mapping to investigate how studies on robotics, in the educational context, have used electronic devices based on free hardware. Among the obtained results, it was observed that of the 73 relevant studies, 40 were actually classified as robotics, while 13 have potential for this same context. On the other hand, 20 studies were better classified as electronic.
\end{abstract}

Resumo. Os dispositivos eletrônicos, baseados em hardware livre, têm sido amplamente utilizados em trabalhos sobre robótica no contexto educacional. No entanto, observa-se que alguns estudos confundem os conceitos de "eletrônica" com "robótica". Logo, este trabalho tem como objetivo o desenvolvimento de um mapeamento sistemático para investigar como os estudos sobre robótica, no contexto educacional, têm usado os dispositivos eletrônicos baseados em hardware livre. Dentre os resultados obtidos, observou-se que dos 73 estudos relevantes, 40 foram classificados de fato como robótica, enquanto 13 possuem potencial para este mesmo contexto. Por outro lado, 20 estudos foram melhor classificados como eletrônica.

\section{Introdução}

De acordo com Chitolina et al. (2016), a robótica aplicada ao contexto educacional possibilita ao aluno vivenciar a aprendizagem de forma mais significativa, ao associar o conhecimento teórico ao prático. No contexto da Computação, a robótica incide como uma tecnologia que permite ao estudante assimilar conceitos abstratos, a partir de práticas para a criação, construção e programação de robôs [D'Abreu et al. 2011, Galvão et al. 2016, Miranda \& Suanno 2012]. Além disso, atividades que antes eram consideradas onerosas na programação (ex.: teste de mesa ${ }^{1}$ ), passam a ter um novo significado para o aluno, devido à facilidade de identificar os erros de código quando executados em um robô [Chitolina et al. 2016].

\footnotetext{
${ }^{1} \mathrm{O}$ teste de mesa consiste na simulação manual (linha a linha) da execução de um programa.
} 
Um robô pode ser definido como um dispositivo formado por componentes eletromecânicos, capazes de realizar uma ou mais tarefas de forma autônoma, ou seja, sem a necessidade de intervenção humana [Gonçalves 2007]. De acordo com Martins et al. (2012), os robôs podem ser classificados como: móveis, com capacidade de se locomover; ou manipuladores que, em geral, assemelham-se a braços robóticos para manusear objetos ou materiais.

Estudos na literatura destacam a importância dos robôs como instrumentos da robótica para apoiar o desenvolvimento de habilidades e competências relacionadas ao pensamento computacional (ex.: raciocínio lógico, abstração, decomposição), que são essenciais para as diversas áreas das Ciências [Avila et al. 2017]. No entanto, para que esse recurso tenha maior alcance nas escolas, em especial nas instituições públicas, pesquisadores têm buscado alternativas para o desenvolvimento de soluções a baixo custo [Costella et al. 2017]. Nesse contexto, têm-se os dispositivos eletrônicos baseados em hardware livre, por exemplo, o Arduino [Banzi e Evans 2014] que consiste em uma placa eletrônica, disponível em diferentes modelos (ex.: Uno, Mega, Nano, dentre outros), composta por circuitos e um processador AVR. Essas placas podem ser vendidas isoladamente ou por meio de kits formados por diversos componentes eletrônicos (ex.: LEDs, sensores, motores).

Apesar do amplo uso desses dispositivos nas pesquisas sobre a robótica na educação [Costella et al. 2017, Galvão et al. 2016, Martins et al. 2012], observa-se que alguns trabalhos confundem os conceitos de eletrônica com robótica. Por exemplo, estudos cujo propósito é o desenvolvimento de um circuito composto por LEDs para ligar luzes, não deveriam utilizar a terminologia "robótica". O uso desse termo só fará sentido, se o dispositivo construído for um robô. Logo, este trabalho tem como objetivo o desenvolvimento de um mapeamento sistemático para investigar como os estudos sobre robótica, no contexto educacional, têm utilizado os dispositivos eletrônicos baseados em hardware livre.

Antes de iniciar o desenvolvimento deste mapeamento, foi realizada uma busca por outros trabalhos de revisões e/ou mapeamentos sistemáticos da literatura com propósito similar. No entanto, os estudos encontrados tinham outros propósitos, por exemplo, verificar o interesse da comunidade brasileira sobre o uso da robótica na educação [Neto et al. 2015], investigar o uso da robótica para desenvolvimento do pensamento computacional [Avila et al. 2017] e realizar um levantamento dos laboratórios remotos de robótica [Almeida e Netto 2015].

Este artigo está organizado em seis seções. A Seção 1 apresentou o contexto, a motivação e o objetivo do trabalho. Na sequência, a Seção 2 descreve o processo de condução do mapeamento sistemático. Na Seção 3, os resultados do mapeamento são mostrados com base nas respostas às questões de pesquisa. Na Seção 4 , os resultados são discutidos com alguns direcionamentos à comunidade da Informática na Educação. $\mathrm{Na}$ Seção 5 têm-se as ameaças à validade e, finalmente, na Seção 6, as conclusões são apresentadas.

\section{Mapeamento Sistemático}

O método de pesquisa utilizado neste trabalho baseou-se em um mapeamento sistemático, que consiste em um tipo de estudo secundário usado para coletar trabalhos 
publicados na literatura (estudos primários), com o intuito de obter o maior número de informações sobre um tópico central de pesquisa [Petersen et al. 2008]. O processo de condução deste mapeamento foi dividido em cinco etapas: (1) definição das questões de pesquisa, (2) definição da string de busca, (3) definição dos critérios de inclusão e exclusão, (4) seleção dos estudos primários relevantes, (5) apresentação e discussão dos resultados.

\subsection{Questões de Pesquisa}

Inicialmente, foram definidas as questões de pesquisa com o objetivo de verificar as características dos "objetos-proposta", apresentados nos artigos sobre o uso da robótica no contexto educacional. O termo "objeto-proposta" é usado neste trabalho para referenciar, por exemplo, dispositivos robóticos (ex.: robô móvel ou manipulador), circuitos eletrônicos (ex.: circuito Arduino), dentre outros.

- QP01 - Quais são os dispositivos eletrônicos, baseados em hardware livre, utilizados nos objetos-proposta?

A questão de pesquisa QP01 investiga quais dispositivos eletrônicos, baseados em hardware livre, têm sido usados nos estudos de robótica, no contexto educacional, para apoiar a concepção, construção e/ou programação dos objetos-proposta.

- QP02 - O objeto-proposta possui autonomia?

A questão de pesquisa QP02 verifica quais objetos-proposta são capazes de realizar suas tarefas de forma independente, ou seja, sem a necessidade de intervenção humana após o início de sua execução.

- QP03 - O objeto-proposta é eletromecânico?

A questão de pesquisa QP03 analisa quais são os objetos-proposta que utilizam em sua estrutura a combinação de componentes eletroeletrônicos (ex.: motores, sensores) e componentes mecânicos (ex.: rodas, eixos).

- QP04 - O objeto-proposta é móvel ou manipulador?

A questão de pesquisa QP04 verifica se os objetos-proposta são móveis, ou seja, se são capazes de se locomover (ex.: em terra, na água, no ar); ou manipuladores, que em geral se assemelham ao braço humano.

- QP05 - O objeto-proposta é um robô?

A questão de pesquisa QP05 identifica que objetos-proposta são considerados robôs, ou seja, que atendem aos requisitos de serem autônomos, eletromecânicos, e do tipo móvel ou manipulador.

- QP06 - O objeto-proposta é recomendado para uso no contexto da robótica?

A questão de pesquisa QP06 investiga quais objetos-proposta podem de fato ou possuem potencial para serem usados na concepção, construção e/ou programação de robôs.

\subsection{Definição da String de busca}

O passo seguinte consistiu na construção da string de busca que foi formada pelos seguintes termos: robótica OU robô OU robotic OU robot. As buscas abrangeram 
artigos publicados no período de 2010 a 2019, nos anais de cinco conferências brasileiras da Informática na Educação: SBIE (Simpósio Brasileiro de Informática na Educação), WIE (Workshop de Informática na Escola), WCBIE (Workshop do Congresso Brasileiro de Informática na Educação), WEI (Workshop sobre Educação em Computação) e WRE (Workshop de Robótica na Educação).

\subsection{Definição dos Critérios de Inclusão e Exclusão}

Na sequência, foram definidos os critérios de inclusão e exclusão para serem aplicados no total de artigos candidatos selecionados na primeira e segunda fase do processo de seleção.

- Critério de Inclusão: (1) estudos sobre robótica no contexto educacional que utilizam a eletrônica baseada em hardware livre.

- Critérios de Exclusão: (1) estudos cujo idioma não esteja em inglês ou português; (2) estudos secundários publicados na literatura; (3) estudos que não especificam o objeto-proposta; (4) estudos que apenas analisam softwares, plataformas e kits de robótica, e arquitetura dos robôs educacionais; (5) estudos que não focam no ensino e/ou aprendizagem de robótica; (6) estudos que têm como proposta a apresentação de ambientes de programação para ensino de robótica.

\subsection{Seleção dos Estudos Primários Relevantes}

O processo de seleção dos estudos primários relevantes foi realizado em três etapas apresentadas na Figura 1.

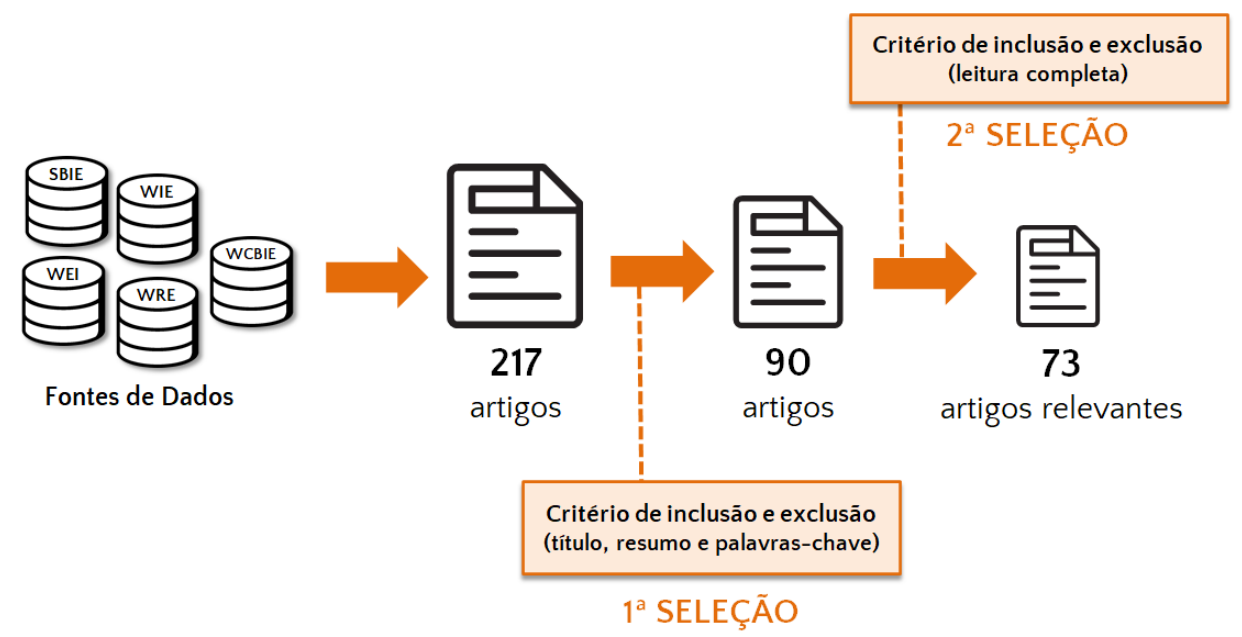

Figura 1. Etapas do processo de seleção.

A primeira etapa exibe o total de 217 artigos retornados pelas cinco fontes de dados. Estes artigos foram divididos aleatoriamente entre os autores e submetidos aos critérios de inclusão e exclusão nas seções de título, resumo e palavras-chave. Na segunda etapa, restaram 90 artigos que foram novamente divididos aleatoriamente pelos autores para a leitura completa dos trabalhos e mais uma vez aplicados os critérios de inclusão e exclusão. Por fim, na terceira etapa restaram 73 estudos primários relevantes.

A Tabela 1 apresenta o número de artigos, por fonte de dados e número de artigos excluídos, após a primeira e segunda seleção. Vale ressaltar que o motivo da 
grande maioria dos artigos terem sido excluídos na primeira seleção, foi por utilizar outros kits de robótica cuja eletrônica não se baseia em hardware livre (ex.: Lego Mindstorms).

Tabela 1. Número de artigos por fonte de dados.

\begin{tabular}{|l|c|c|c|c|c|c|c|}
\hline & SBIE & WIE & WCBIE & WEI & WRE & Artigos Excluídos & Total \\
\hline Inicial & 44 & 58 & 27 & 20 & 68 & - & 217 \\
\hline Primeira seleção & 11 & 24 & 16 & 11 & 28 & 127 & 90 \\
\hline Segunda seleção & 10 & 23 & 13 & 7 & 20 & 17 & 73 \\
\hline
\end{tabular}

\section{Resultados}

Nesta seção são apresentadas as respostas às questões de pesquisa apresentadas na Seção 2.1 com base nos 73 estudos $^{2}$ selecionados como relevantes. Vale ressaltar que as citações sinalizadas com um asterisco $\left(^{*}\right)$ fazem parte da lista de estudos primários obtidos a partir do mapeamento.

QP01 - Quais são os dispositivos eletrônicos, baseados em hardware livre, utilizados nos objetos-proposta?

Foram identificados quatro dispositivos eletrônicos baseados em hardware livre utilizados na concepção, construção e/ou programação dos objetos-proposta. São eles: (i) Raspberry Pi, (ii) Br-Gogo, (iii) BeagleBone Black e (iv) Arduino. Os dispositivos Raspberry Pi, Br-Gogo e BeagleBone Black foram empregados, respectivamente, nos trabalhos *Rodrigues et al. (2015), *D'Abreu et al. (2011) e *Andrade et al. (2015) . Finalmente, os $\mathbf{7 0}$ estudos restantes fizeram uso da placa Arduino de diferentes modelos (ex.: Uno, Nano, Mega, dentre outros).

QP02 - O objeto-proposta possui autonomia?

Neste trabalho, foram considerados como autônomos os objetos-proposta que executam os comandos embarcados de forma independente, ou seja, sem a necessidade de intervenção humana para realizar uma tarefa. Logo, dos 73 artigos selecionados, foi constatado que em 19 estudos os objetos-proposta não possuíam autonomia, pelos seguintes motivos: ou por não deixar essa informação clara no artigo [*Silva \& Almeida 2012] ou pelo fato do objeto-proposta necessitar de influência humana para realizar alguma ação. Por exemplo, acionar um interruptor de luz [*Monteiro et al. 2016], movimentar um guincho [*Pancieri et al. 2019], dentre outros.

Por outro lado, nos 54 estudos restantes foi verificada a presença de objetosproposta que possuíam autonomia para, por exemplo, desviar de obstáculos [*Zanetti \& Oliveira 2015], mapear a superfície de uma região [*Rodrigues et al. 2015], desenhar em uma lousa [*Ferreira et al. 2019a, *Cerci et al. 2017], irrigar solos de forma inteligente [*Reinoso et al. 2017], simular um semáforo de trânsito [*Silva \& Javaroni 2018], ligar um cubo de LEDs [*Bessa et al. 2017], dentre outros.

QP03 - O objeto proposto é eletromecânico?

\footnotetext{
${ }^{2} \mathrm{~A} \quad$ lista dos 73 estudos relevantes encontra-se disponível no link https://clausiusreis.github.io/mapeamento_robotica/pdf/referencias.pdf.
} 
Dos 73 artigos selecionados, em 20 estudos os objetos-proposta não são eletromecânicos, ou seja, utilizam em sua construção apenas componentes eletrônicos. Por exemplo, LEDs para simular as luzes de um semáforo de trânsito [*Silva \& Javaroni 2018, *Silva \& Oliveira 2019]; sensores para controlar o nível de água da chuva e umidade do solo em um sistema de irrigação inteligente [*Reinoso et al. 2017], dentre outros.

Por outro lado, nos $\mathbf{5 3}$ estudos restantes os objetos-proposta são eletromecânicos, ou seja, utilizam em sua estrutura a combinação de componentes eletrônicos e mecânicos. Exemplos de objetos-proposta com essa estrutura podem ser encontrados em *Ferreira et al. (2019a), *Cerci et al. (2017), *Costella et al. (2017), dentre outros.

QP04 - O objeto-proposta é móvel ou manipulador?

Dos 73 artigos selecionados, em 20 estudos os objetos-proposta não foram classificados como do tipo móvel ou manipulador. Neste contexto, têm-se os trabalhos que simulam, por exemplo, um semáforo de trânsito [*Silva \& Javaroni 2018, *Silva \& Oliveira 2019], um sistema de irrigação inteligente [*Reinoso et al. 2018], cubo de LEDs [*Bessa et al. 2017], dentre outros.

Por outro lado, nos $\mathbf{5 3}$ estudos restantes os objetos-propostas foram classificados como do tipo móvel ou manipulador. Como exemplos de objetos-proposta móveis têm-se: robô com seis pernas [*Andrade et al. 2015]; robô com rodas [*Flores et al. 2018, *Silva et al. 2018] e cabeça [*Martins et al. 2012]; robô desenhador de lousa [*Ferreira et al. 2019a, *Cerci et al. 2017], dentre outros. Como exemplos de objetosproposta manipuladores tem-se: braço robótico [*Costella et al. 2017] e manipulador robótico no formato de luva [*Vasconcellos et al. 2015].

QP05 - O objeto-proposta é um robô?

Neste trabalho um objeto-proposta será considerado um robô se for: autônomo, eletromecânico e do tipo móvel ou manipulador. Logo, dos 73 estudos selecionados em 40 artigos os objetos-proposta foram classificados como robôs por atender aos três requisitos anteriores. Por exemplo: braço robótico [*Costella et al. 2017], robô de competição [*Santos et al. 2019, *Martins et al. 2012], robô desenhador de lousa [*Ferreira et al. 2019a, ${ }^{*}$ Cerci et al. 2017], robô humanoide [*Amorim et al. 2016], robô de resgate [*Angonese et al. 2012], dentre outros.

Por outro lado, nos $\mathbf{3 3}$ artigos restantes os objetos-proposta não foram classificados como robô, por não atender a pelo menos um dos três requisitos: possuir autonomia, ser eletromecânico e/ou ser do tipo móvel/manipulador. Nesse contexto tem-se o simulador de semáforo de trânsito [*Silva \& Javaroni 2018, *Silva \& Oliveira 2019], sistema de irrigação inteligente [*Reinoso et al. 2017], cubo de LEDs [*Bessa et al. 2017], tapete alfabético [*Ferreira et al. 2019], dentre outros.

QP06 - O objeto-proposta é recomendado para uso no contexto da robótica?

Os trabalhos recomendados para uso no contexto da robótica são aqueles que apresentam soluções que possam ser usadas (de fato ou com potencial) na concepção, construção e/ou programação de robôs. Logo, dos 73 estudos selecionados, em 53 artigos os objetos-proposta são indicados para uso no contexto da robótica, enquanto 
20 artigos não são. Mais detalhes sobre os resultados para essa questão de pesquisa são apresentados na Seção 4.

\section{Discussão dos Resultados}

Conforme mostrado na Figura 2, foram identificados quatro fluxos diferentes para os 73 estudos considerados relevantes para este trabalho: dois em que o objeto-proposta é recomendado para uso no contexto da robótica e dois para uso no contexto da eletrônica.

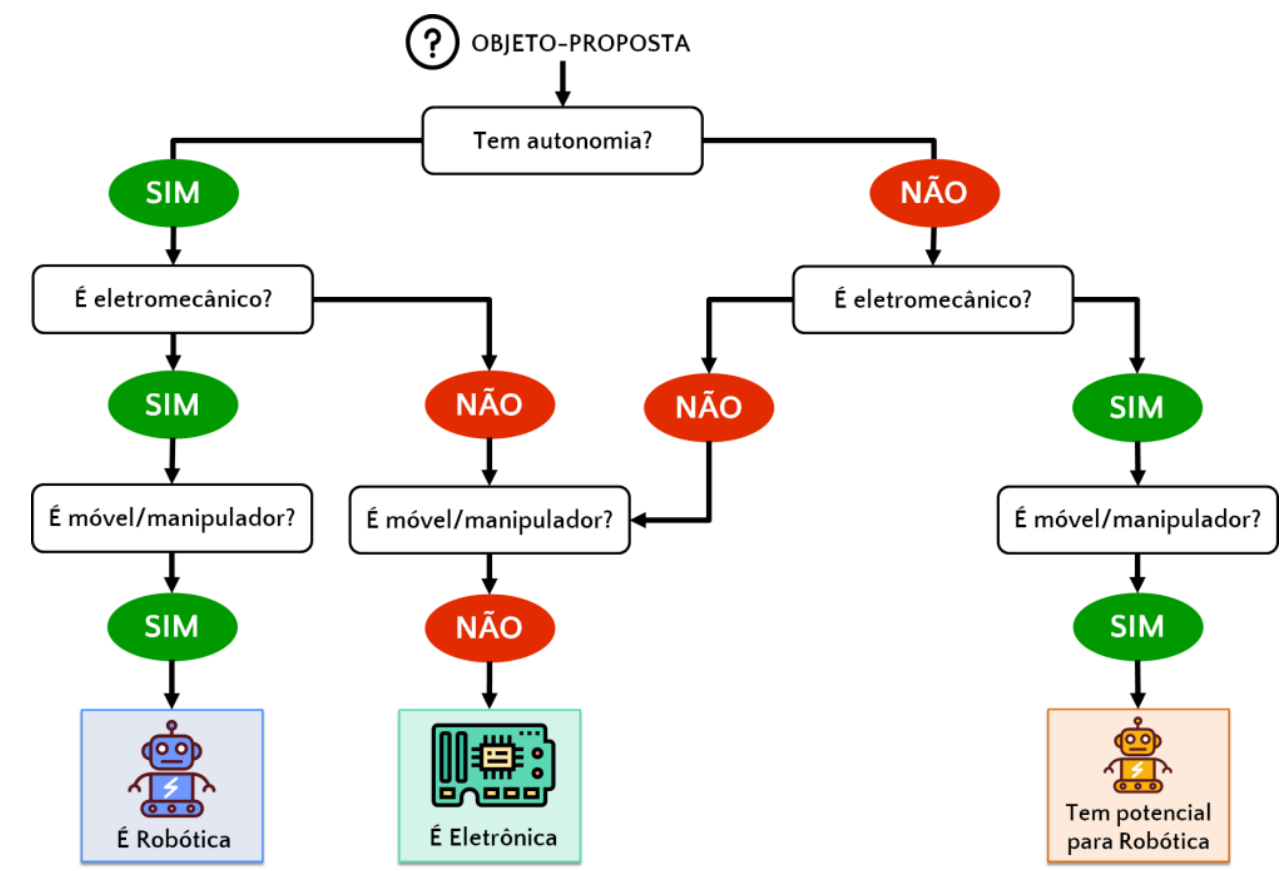

Figura 2. Fluxograma para classificação do objeto-proposta como robótica ou eletrônica.

O primeiro e quarto fluxos caracterizam, respectivamente, os trabalhos que são robótica ou que possuem potencial para uso na robótica. No primeiro fluxo, os trabalhos que são robótica contemplam os objetos-proposta que: (1) possuem autonomia, (2) são eletromecânicos e (3) são móveis ou manipuladores. Logo, pode-se dizer que os objetos-proposta são de fato um robô. Nesse contexto, foram identificados 40 estudos que propuseram, por exemplo, robô de competição [*Santos et al. 2019, *Martins et al. 2012], robô desenhador de lousa [*Ferreira et al. 2019a, ${ }^{*}$ Cerci et al. 37], robô humanoide [*Amorim et al. 2016], robô de resgate [*Angonese et al. 2012], dentre outros.

O quarto fluxo mostrado na Figura 2 reflete os trabalhos com potencial para uso na robótica, ou seja, são aqueles em que os objetos-proposta: (1) não possuem autonomia, (2) são eletromecânicos e (3) são móveis ou manipuladores. Neste caso, os objetos-proposta não são de fato um robô, pelo fato de não atenderem ao requisito de autonomia. No entanto, dependendo da abordagem utilizada pelos estudos, a adição da característica de autonomia pode ser contornada. Por exemplo, nos estudos que apresentam apenas a construção de robôs [*Silva et al. 2018, *Silva \& Almeida 2012], não foi possível atestar a autonomia do robô. Nesse sentido, os autores poderiam, por 
exemplo, indicar a presença de componentes eletrônicos (ex.: sensores ultrassônicos) e mencionar que a programação deve assegurar a autonomia do robô (ex.: desviar de obstáculos de forma independente). Por outro lado, no trabalho de *Moreira et al. 2015, o robô executa suas ações a partir de um controle remoto de televisão manipulado por um usuário humano. Neste caso, o estudo foi classificado como com potencial para robótica, pois poderia ser feita a substituição do controle remoto por sensores eletrônicos para assegurar a autonomia do robô. Vale ressaltar que dos 73 estudos selecionados, 13 artigos se enquadraram nesse contexto.

De acordo com a Figura 2, o segundo e terceiro fluxos caracterizam os trabalhos recomendados para uso no contexto da eletrônica baseada em hardware livre. No segundo fluxo, estão os trabalhos em que os objetos-proposta: (1) são autônomos, (2) não são eletromecânicos e (3) não são móveis ou manipuladores. Neste caso, os objetos-proposta não atendem a duas características dos robôs. A alternativa de transformar, por exemplo, os circuitos eletrônicos em eletromecânicos seria inviável, pois acarretaria na mudança de toda estrutura do objeto-proposta. Nesse contexto, foram identificados 14 artigos. Por exemplo, simulador de semáforo de trânsito [*Silva \& Javaroni 2018, *Silva \& Oliveira 2019, *Silva et al. 2016], sistema de irrigação inteligente [*Reinoso et al. 2017], cubo de LEDs [*Bessa et al. 2017], dentre outros.

Por fim, no terceiro fluxo, estão os trabalhos em que os objetos-proposta: (1) não são autônomos, (2) não são eletromecânicos e (3) não são móveis ou manipuladores. Neste caso, os objetos-proposta não atendem a nenhum dos requisitos de um robô. Logo, assim como no caso anterior (fluxo 2), a recomendação é que os trabalhos com essas características não sejam usados no contexto da robótica e sim da eletrônica. Nesse contexto, foram identificados seis trabalhos. Por exemplo, tapete alfabético [*Ferreira et al. 2019], montagem de circuitos com LEDs e LDR [*Sobreira et al. 2016], luva para deficiente visual [*Silva et al. 2016], dentre outros.

Devido à restrição de espaço, não foi possível incluir as citações dos 73 trabalhos na discussão dos resultados. Logo, para que os leitores tenham acesso a classificação de todos os artigos, foi elaborada uma visualização interativa (Figura 3), que pode ser acessada no link: https://clausiusreis.github.io/mapeamento_robotica/.

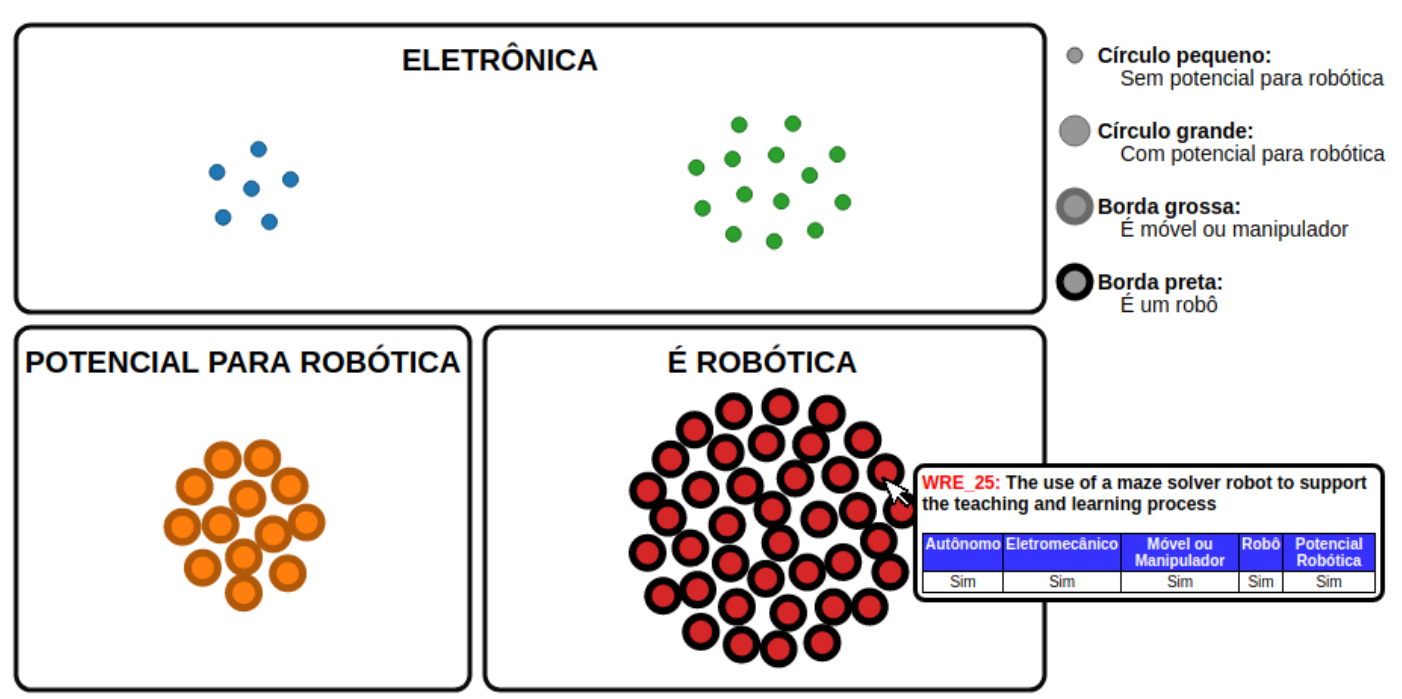

Figure 3. Visualização interativa para acessar as informações dos 73 estudos. 
Ao passar o cursor sobre as amostras (círculos) da visualização interativa, é possível acessar as informações dos trabalhos. Além disso, para ter acesso ao artigo original, o leitor deve clicar duas vezes na amostra desejada. Essa visualização agrupa os trabalhos por cores com base nos quatro fluxos apresentados na Figura 2.

- Vermelho: é autônomo, é eletromecânico, é móvel ou manipulador;

- Verde: é autônomo, não é eletromecânico, não é móvel ou manipulador;

- Laranja: não é autônomo, é eletromecânico, é móvel ou manipulador;

- Azul: não é autônomo, não é eletromecânico, não é móvel ou manipulador.

\section{Ameaças à Validade}

Este mapeamento sistemático apresentou algumas limitações que podem ter influenciado os resultados. A primeira consiste na divisão dos artigos entre os autores para análise e extração dos dados. Apesar de todos os autores terem observado os critérios de inclusão e exclusão e discutido suas dúvidas em reuniões periódicas com a equipe, algumas interpretações subjetivas podem ter influenciado a categorização dos resultados. Outra limitação está relacionada à classificação dos 73 trabalhos finais quanto ao conceito de autonomia. Os autores entendem que assegurar a autonomia de um dispositivo robótico e/ou eletrônico pode variar de contexto para contexto. Logo, nesse sentido, foram classificados como autônomos quaisquer dispositivos que executem os comandos embarcados de forma independente, não fazendo diferenciação, por exemplo, entre a execução de um semáforo de trânsito simples [*Silva \& Javaroni 2018] com um semáforo de trânsito inteligente [*Silva \& Oliveira 2019].

\section{Conclusão}

Este trabalho teve como objetivo o desenvolvimento de um mapeamento sistemático para investigar o uso do termo "eletrônica baseada em hardware livre" nos estudos sobre robótica voltados para o contexto educacional. Para a realização desta pesquisa, cinco fontes de dados brasileiras foram consultadas e 217 artigos analisados. Desse total, 90 atenderam aos critérios de inclusão e exclusão e 73 foram considerados relevantes para responder às questões de pesquisa.

Os resultados mostraram que do total de 73 trabalhos, $\mathbf{4 0}$ realizaram pesquisas para apoiar a concepção, construção e/ou programação de soluções que são de fato robôs, sendo, portanto, adequadas para uso no contexto da robótica aplicada à educação. Desse conjunto, vale ressaltar que 95\% (38 estudos) utilizaram o Arduino como eletrônica baseada em hardware livre. Além disso, 13 estudos demonstraram ter potencial para serem usados no contexto da robótica, desde que fosse assegurada a autonomia dos robôs em trabalhos futuros. Por outro lado, foi observado que $\mathbf{2 0}$ estudos não são robótica e também não possuem potencial para uso na robótica, sendo melhor classificados como eletrônica no contexto educacional. Desse conjunto (20 artigos), vale destacar que $100 \%$ dos trabalhos fizeram uso do Arduino como dispositivo eletrônico baseado em hardware livre.

Como contribuição, espera-se que o fluxograma apresentado na Figura 2, possa ser usado pela comunidade da Informática na Educação para a classificação adequada dos seus trabalhos como robótica ou eletrônica. Como trabalho futuro, sugere-se a 
IX Congresso Brasileiro de Informática na Educação (CBIE 2020)

Anais do XXXI Simpósio Brasileiro de Informática na Educação (SBIE 2020)

ampliação desse fluxograma com a adição de novos fluxos não abordados neste trabalho.

\section{Referências}

Almeida, T. O. \& Netto, J. F. de M. (2015). "Robótica Pedagógica Aplicada ao Ensino de Programação: Uma Revisao Sistemática da Literatura", Anais do XXVI Simpósio Brasileiro de Informática na Educação, p.597-606.

Avila, C., Simone, C., Bordini, A., \& Marques, M. (2017). "O Pensamento Computacional por meio da Robótica no Ensino Basico - Uma Revisão Sistemática”, Anais do XXVIII Simpósio Brasileiro de Informática na Educação, p. 82-91.

Banzi, M., Michael S. (2014). Getting started with Arduino: the open source electronics prototyping platform. Make Books - Imprint of: O'Reilly Media, Sebastopol, CA.

Chitolina, R. F., Noronha, F. P. T., \& Backes, L. (2016). A Robótica Educacional como tecnologia potencializadora da aprendizagem: das ciências da natureza às ciências da computação. Educação, Formação \& Tecnologias, p. 57-65.

Costella, L., Trentin, M., Amarante, V., \& Teixeira, A. (2017). "Construção de ambiente de ensino de robótica remota: Democratizando o desenvolvimento do pensamento computacional em alunos da educação básica". Anais do XXVIII Simpósio Brasileiro de Informática na Educação, p. 354- 363.

D'Abreu, J. V. V., Mirisola, L. G., \& Ramos, J. J. (2012). “Ambiente de robótica pedagógica com Br_GOGO e computadores de baixo custo: Uma contribuição para o ensino médio", Anais do XXII Simpósio Brasileiro de Informática na Educação, Aracaju - SE, p. 100-109.

Galvão, A. P., Thomas, A., \& Pereira, P. M. P (2016). “Aplicação do Arduíno através de Robótica Educacional para apoio ao Ensino-Aprendizagem de Estudantes do Ensino Médio no Oeste do Pará", Anais do VII WRE, Recife - PE, p. 107-111.

Gonçalves, P. C. (2007). Protótipo de um Robô Móvel de Baixo Custo para Uso Educacional, Dissertação de Mestrado, Universidade Estadual de Maringá (Dissertação de Mestrado), Maringá-PA, 87p..

Martins, F. N., Oliveira, H. C., \& Oliveira, G. F. (2012). "Robótica como meio de promoção da interdisciplinaridade no ensino profissionalizante", Anais do III Workshop de Robótica Educacional, Natal - RN, p. 1-11.

Miranda, J. R. \& Suanno, M. V. (2012). "Robótica na escola: ferramenta pedagógica inovadora", Anais do III Workshop de Robótica Educacional, Natal - RN, p. 1-11.

Neto, R. P. B., Rocha, D. P., Santana, A. M., \& Souza, A. A. de S. (2015). "Robótica na Educação: Uma Revisão Sistemática dos Ultimos 10 anos", Anais do XXVI Simpósio Brasileiro de a Informática na Educação, Maceió - AL, p. 386 - 393.

Petersen, K., Feldt, R., Mujtaba, S., Mattsson, M. (2008). "Systematic mapping studies in software engineering", Proceedings of the 12th international conference on evaluation and assessment in software engineering, p. 68-77.Ciudad Real, Spain: ACM: British Computer Society. 